\title{
Fuzzy soft set based decision making: a novel alternative approach
}

\author{
José Carlos R. Alcantud ${ }^{1}$ \\ ${ }^{1}$ Facultad de Economía y Empresa and IME, University of Salamanca, 37007 Salamanca, Spain
}

\begin{abstract}
We take advantage of a recent development in Social Choice in order to propose a new algorithm for the prioritization of objects characterized by fuzzy soft sets. It benefits from the performance of an endogenous scoring rule recently proposed by Herrero [1]. Our procedure constructs a "comparison matrix" and grades the objects according to the corresponding component of an eigenvector associated with its dominant eigenvalue. This eigenvector can be easily calculated because the dominant eigenvalue is computed directly from the size of the tabular representation of the problem.
\end{abstract}

Keywords: Fuzzy soft set, Fuzzy decision making, Comparison table, Endogenous scoring rule, Borda count.

\section{Introduction}

In this paper we revisit the fuzzy soft set based decision making problem as posed by Roy and Maji [2], and subsequently approached by authors like Kong et al. [3], Feng et al. [4] and Alcantud [5].

Since its introduction by Zadeh [6] there is a vast literature on fuzzy set theory and their applications, as well as succesful variations intending to capture subjectivity, uncertainty, imprecision of the appraisals, ... in order to better handle practical situations.

In particular, the theory of soft sets was initiated by Molodtsov [7] who showed its applicability to several fields. He also established some fundamental results, for example, that the models by fuzzy sets and soft sets are not independent. Alcantud [8] proves further relationships among these concepts and others. Further developments appear in Maji et al. [9] and Aktaş and Çă̆man [10] among others. Contributions like Ali [11], Feng et al. [12] and Feng et al. [13] improve our knowledge about connections among soft sets, fuzzy sets and other soft computing models.

Subsequently, Maji, Biswas and Roy [14] introduce fuzzy soft sets, the main object of analysis of our contribution. Wang, Li and Chen [15] introduce hesitant fuzzy soft sets. Han et al. [16] and Zou and Xiao [17] are concerned with incomplete soft sets. Jiang et al. [18] present an extended soft set theory based on description logics.
The pioneering approach to deal with fuzzy soft set based decision making situations is Roy and Maji [2]. It is a useful complement to the standard solutions for soft set based decision making problems (cf., Maji, Biswas and Roy [19], Çă̆man and Enginoğlu [20] and Feng and Zhou [21]). Their procedure relies on the computation of a comparison matrix which permits to attach scores to the alternatives or objects characterized by fuzzy soft sets. The work by Alcantud [5] intends to overcome its tendency to get ties, by the recourse to a different computation of the comparison matrix. In a different vein Feng et al. [4] incorporate subjectivity into the analysis of this issue. They develop an adjustable method based on level soft sets under which the optimal choice is dependent on the selected level soft sets.

In this contribution we address the problem posed in [2] but we do not follow the aforementioned interesting lines of inspection. Instead, we take advantage of a recent proposal in Social Choice that permits to suggest a completely redesigned algorithm for the prioritization of objects characterized by fuzzy soft sets.

More precisely, our algorithm constructs a different "comparison matrix" which benefits from the analysis of an endogenous scoring rule recently proposed by Herrero [1]. It is a positive matrix whose cells are Borda counts (in its diagonal) and Condorcet dominations (off its diagonal). Then our procedure grades the objects according to the corresponding component of an eigenvector associated with the dominant eigenvalue of the matrix. The investigation by Herrero permits to import interpretations, characteristics and relevant features of this solution. Thus for example, the dominant eigenvalue of the matrix is trivially calculated from the size of the corresponding tabular specification.

The structure of the problem ensures that our procedure ends in a final decision in all circumstances. Therefore, contrary to the adjustable methodology our algorithm produces well-defined and uniquely determined optimal decisions. And it inherits other virtues of the endogenous scoring rule in Herrero [1]. This means a further benefit with respect to the common score-based approach in Roy and Maji [2] and Alcantud [5].

This paper is structured as follows. In Section 2 we recall some preliminary definitions, present the problem, and review the most relevant literature. 
In particular, in subsection 2.3 we describe some solutions to the problem under inspection in previous related approaches. In Section 3 we explain the construction and properties of the tool from Social Choice whose methodology we intend to import. Then we propose a novel solution for our problem in the form of an algorithm. Section 4 contains an extensive example that illustrates the methodology that we endorse here. In Section 5 we compare our proposal with previous procedures. We conclude and mention other related lines of research in Section 6 .

\section{The problem and a critical review of existing solutions}

This Section is devoted to establish our framework, present the problem, and review the most relevant literature. We also examine some solutions to the problem of fuzzy soft set based decision making provided by the literature, which rely on the use of scores.

\subsection{Soft sets and fuzzy soft sets}

In order to define our framework, henceforth $U$ denotes a universe of objects and $E$ denotes a universal set of parameters.

The origin of our investigation is the following concept:

Definition 1 (Molodtsov [7]) The pair $(F, A)$ is a soft set over $U$ when $A \subseteq E$ and $F: A \longrightarrow \mathcal{P}(U)$, where $\mathcal{P}(U)$ denotes the set of all subsets of $U$.

A soft set over $U$ is regarded as a parameterized family of subsets of the universe $U$, the set $A$ being the parameters. For each $e \in A, F(e)$ may be considered as the set of $e$-approximate elements of the soft set $(F, A)$.

Maji, Bismas and Roy [9] develop the original analysis of soft sets further. They define concepts like soft subsets and supersets, soft equalities, intersections and unions of soft sets, among others. Feng and Li [22] give a systematic study on several types of soft subsets and various soft equal relations induced by them.

Definition 2 below has been subsequently proposed and investigated in order to model increasingly general situations:

\section{Definition 2 (Maji, Biswas and Roy [14])}

The pair $(F, A)$ is a fuzzy soft set over $U$ when $A \subseteq E$ and $F: A \longrightarrow \mathbf{F S}(U)$, where $\mathbf{F S}(U)$ denotes the set of all fuzzy sets on $U$.

Clearly, every soft set can be considered as a fuzzy soft set.

When both $U$ and $A$ are finite (as is common to most real applications) fuzzy soft sets can be represented either by matrices or in tabular form. Rows are attached with objects in $U$, and columns are

\begin{tabular}{|c|c|c|c|c|c|c|c|}
\hline & $p_{1}$ & $p_{2}$ & $p_{3}$ & $p_{4}$ & $p_{5}$ & $p_{6}$ & $p_{7}$ \\
\hline$o_{1}$ & 0.3 & 0.1 & 0.4 & 0.4 & 0.1 & 0.1 & 0.5 \\
$o_{2}$ & 0.3 & 0.3 & 0.5 & 0.1 & 0.3 & 0.1 & 0.5 \\
$o_{3}$ & 0.4 & 0.3 & 0.5 & 0.1 & 0.3 & 0.1 & 0.6 \\
$o_{4}$ & 0.7 & 0.4 & 0.2 & 0.1 & 0.2 & 0.1 & 0.3 \\
$o_{5}$ & 0.2 & 0.5 & 0.2 & 0.3 & 0.5 & 0.5 & 0.4 \\
$o_{6}$ & 0.3 & 0.5 & 0.2 & 0.2 & 0.4 & 0.3 & 0.3 \\
\hline
\end{tabular}

Table 1: Tabular representation of the fuzzy soft set $(S, P)$ in Roy and Maji [2].

attached with parameters in $A$. In the particular instance of a soft set, these representations are binary: all cells are either 0 or 1 . As an example, Table 1 puts forward the tabular representation of the fuzzy soft set $(S, P)$ in Roy and Maji [2]. It is used by Alcantud [5] in order to contrast his proposal with other existing solutions.

When a soft set $(F, A)$ is represented in matrix form through the matrix $\left(h_{i j}\right)_{i, j}$ then the choice value of an object $h_{i} \in U$ is $c_{i}=\sum_{j} h_{i j}$. According to Maji, Biswas and Roy [19, Subsection 3.4], a suitable choice is made when the selected object $h_{k}$ verifies $c_{k}=\max _{i} c_{i}$. In other words, objects that maximize the choice value are satisfactory outcomes of this soft set based decision making problem. Alternative solutions include Çă̆man and Enginoğlu [20] and Feng and Zhou [21].

As mentioned above, a list of different approaches to fuzzy soft set based decision making includes the references Roy and Maji [2], Kong et al. [3], Feng et al. [4] and Alcantud [5]. We proceed to survey their main characteristics and differences below in subsection 2.2.

\subsection{Fuzzy soft set based decision making: a review of the literature}

Because of the subjective or humanistic nature of the problem of fuzzy soft set based decision making, which replicates the situation of soft set based decision making that we have described above, it comes as no surprise that several approaches have been proposed to contribute to the controversial and complex issue.

Roy and Maji [2] first proposed an algorithm to prioritize alternatives or objects characterized by fuzzy soft sets. A different procedure is given by Kong et al. [3]. Although these authors claim that the Roy and Maji's algorithm is incorrect on the grounds of a single naive example, such presumed "counterexample" does not stand inspection (cf., Feng et al. [4, Subsection 3.2]). Furthermore, the divergence of opinions between [2] and [3] is whether scores or choice values should be used as the criterion for making the optimum decision, and it seems rather arguable whether choice values are adequate in this framework.

In a different vein, Feng et al. [4] incorporate subjectivity in this analysis by proposing an adjustable 
method based on level soft sets. In their approach the optimal choice is dependent upon the selected level soft sets, which is a burden for the decisionmaker who has to decide about such previous factor without a suitable methodology.

Alcantud [5] has shown by examples that the aforementioned solutions tend to produce many ties among options. Then he proposes a novel method with two specific targets. Firstly, in line with Roy and Maji's acclaimed proposal it appeals to a Comparison table and produces a unique welldetermined outcome through scores. But secondly, his novel procedure is considerably less inconclusive because it leans on a different way to define that relevant Comparison table that ultimately produces fewer draws.

In a different spirit, Jiang et al. [23, Section 1] conclude that the parameters in the specification of the (fuzzy) soft sets are too simple. They present an extended fuzzy soft set theory that appeals to the concepts of fuzzy description logics to act as the parameters of fuzzy soft sets.

We must also acknowledge that the pioneering Roy and Maji [2] directly state their algorithm for multiobserver data in terms of various sets of parameters, e.g., in the problem of object recognition. The aforementioned Alcantud [5] contributes to this related issue as well. He shows that Roy and Maji's original approach may result into a loss of information along the construction of a resultant fuzzy soft set from the multiobserver information. Consequently he provides an alternative proposal for such purpose under which the impact of the unavoidable loss of information is less sizable. The present study is not intended to take sides in this controversy, thus we directly proceed with the information provided by the resultant fuzzy soft set.

\subsection{Two score-based algorithms that solve the problem}

For the sake of subsequent comparisons, let us recall the characteristics of the two score-based proposals that we have emphasized earlier in subsection 2.2 due to their nice performance.

The application of Roy and Maji's algorithm proceeds as under:

Algorithm 1 ([2]). Roy and Maji's algorithm.

\section{Step 1.}

Input the fuzzy soft set $(F, A)$ on $k$ objects in the form of an input table whose cell $(i, j)$ is denoted $t_{i j}$.

\section{Step 2.}

Construct a $k \times k$ matrix $C=\left(c_{i j}\right)_{k \times k}$ where $c_{i j}$ is the number of parameters for which the membership value of $o_{i}$ is greater or equal than the membership value of $o_{j}$. In other words, $c_{i j}$ is the number of parameters $m$ for which $t_{i m}-$ $t_{j m} \geqslant 0$, or the number of non-negative values in the finite sequence $t_{i 1}-t_{j 1}, t_{i 2}-t_{j 2}, \ldots$

This is Roy and Maji's Comparison matrix.

\section{Step 3.}

For each $i=1, \ldots, k$, compute $r_{i}$ as the sum of the elements in row $i$ of $C$, and $t_{i}$ as the sum of the elements in column $i$ of $C$. Then for each $i=1, \ldots, k$, compute the score $s_{i}=r_{i}-t_{i}$ of object $i$.

\section{Step 4.}

The decision is any object $o_{k}$ that maximizes the score, i.e., any $o_{k}$ such that $s_{k}=$ $\max _{i=1, \ldots, k} s_{i}$.

Example 3 below illustrates the use of Algorithm 1 in a concrete situation. As discussed earlier, in a problem with multiobserver input parameter data set the practitioner must first produce the resultant fuzzy soft set $(F, A)$ either with Roy and Maji's [2] or with Alcantud's [5] methodology.

Alcantud [5] discusses the definition of $C$ in $\mathrm{Al}-$ gorithm 1 at length, presents several demonstrative examples, and states a proposal that overcomes drawbacks of earlier approaches. The main novelty in his proposal regarding Algorithm 1 is in Step 2, i.e., in the definition of the Comparison matrix. Then his algorithm produces scores denoted $S_{i}$ henceforth.

Example 1 In the case of the fuzzy soft set $(S, P)$ represented by Table 1, the algorithm in Alcantud [5] produces the outcome

$$
o_{5} \succ o_{6} \succ o_{3} \succ o_{2} \succ o_{1} \succ o_{4}
$$

(a simplified notation which stands for the prioritization $S_{5}>S_{6}>S_{3}>S_{2}>S_{1}>S_{4}$ of the scores computed by his algorithm). This is fairly similar to the solution by Roy and Maji [2], namely, $o_{5} \succ o_{3} \succ o_{6} \succ o_{2} \succ o_{1} \succ o_{4}$ (which stands for $s_{5}>s_{3}>s_{6}>s_{2}>s_{1}>s_{4}$, the prioritization of scores computed by Algorithm 1).

\section{A new proposal of solution in fuzzy soft set based decision making}

In soft set based decision making, Maji, Biswas and Roy [19, Subsection 3.5] proposed a straightforward way to generalize their decision mechanism by choice values. They propose to impose weights on each choice parameter in order to produce a revised algorithm for computing the optimal decisions. Following that line of exploration, Feng et al. [4] introduce the notion of weighted fuzzy soft sets. Through a direct modification of their original adjustable proposal they discuss some applications to fuzzy soft set based decision making problems. However in both their approaches the weights of the parameters are exogenously given and the researcher has no clue as to which exact procedure can be of help at this point. 
This drawback is reminiscent of a problem in Social Choice. Suppose that a group has to vote on a list of options. An extensively used solution appeals to scoring rules, which attach some exogenously given weights to the alternatives depending upon their position in the individual rankings. Of course the choice of the weights heavily affects the final decision, as is the case of the aforementioned approaches to decision making problems in the context of soft set theory.

In the remaining of this Section we import a recent technique that permits to use endogeneously selected weights in voting theory. Firstly we describe the benefits of such rule. And then we put forward a completely redesigned algorithm for decision making in fuzzy soft set theory that inherits such benefits. Thus the practitioner can avoid deciding on the weights without refusing to their appeal, since the algorithm that we propose makes implicit computation and use of weights.

\subsection{Herrero's endogenous scoring rule}

In Social Choice a previous analysis of which particular scoring rule is adopted should be made when weights are to be attached to the opinions on the options. Quite remarkably, Herrero [1] has proposed an endogenous scoring rule that permits to circumvent this handicap, i.e., a voting rule that relies on endogenosly obtained scores. Such scores are given by the relative support of the options. Herrero explains that the relative support is a vector with positive components. Not only it gives a natural order of the options by the size of their support, but also it measures their worth, power or importance.

Let us proceed with the formal analysis of this endogenous scoring rule. Herrero [1] poses several examples which indicate that her rule neither is a scoring rule, nor coincides with the Borda rule nor is Condorcet consistent. Neverheless it combines the Condorcet and the Borda information in order to naturally induce an order of the options by exploiting the properties of a certain non-negative matrix that Herrero calls the Condorcet-Borda matrix. Its structure is easy to explain, and is described in Step 2 of Algorithm 2 below. Associated with its dominant eigenvalue there must be an eigenvector, the components of which are all positive. Herrero convincingly argues that it is natural to interpret its components as a measure of the relative support of the options, thus it gives a natural prioritization for them. To make things easier, Herrero shows that the dominant eigenvalue requires no calculation since it is trivially computed from the size of the tabular representation of the problem.

\subsection{An approach based on eigenvectors}

In view of the discussion above it seems appropriate to import Herrero's methodology of endogenous scores in order to implement the following novel pro- cedure for the purpose of ranking objects characterized by fuzzy soft sets:

Algorithm 2. New approach based on Herrero's methodology.

\section{Step 1.}

Input the fuzzy soft set on $k$ objects in the form of a $k \times q$ input table whose cell $(i, j)$ is denoted $t_{i j}$.

Step 2.

Construct a $k \times k$ matrix $C=\left(c_{i j}\right)_{k \times k}$ where:

(a) When $i \neq j, c_{i j}$ is the number of parameters for which the membership value of $o_{i}$ is strictly greater than the membership value of $o_{j}$. Thus $c_{i j}$ is the number of parameters $m$ for which $t_{i m}-t_{j m}>0$, or the number of positive values in the finite sequence $t_{i 1}-t_{j 1}, t_{i 2}-t_{j 2}, \ldots$

(b) For each $i, c_{i i}=q(k-1)-m_{i}$ where $m_{i}=$ $\sum_{j \neq i} c_{j i}$ is the sum of the non-diagonal elements in column $i$ of $C$.

This is Herrero's Comparison matrix.

\section{Step 3.}

Compute one eigenvector $H=\left(H_{1}, \cdots, H_{k}\right)$ associated with the dominant eigenvalue of the matrix, namely, $q(k-1)$.

\section{Step 4.}

The decision is any object $o_{k}$ such that $k$ maximizes the component of $H$, i.e., any $o_{k}$ such that $H_{k}=\max _{i=1, \ldots, k} H_{i}$.

The only apparent difficulty in the application of Algorithm 2 is in Step 3. In reality, the computation of eigenvectors for a known eigenvalue is trivial, since it boils down to solving a system of $k$ linear equations. This is computationally simple. Nevertheless standard mathematical software can calculate eigenvectors directly. We emphasize that Herrero's analysis proves that the eigenvalue that is needed is $q(k-1)$.

Algorithm 2 combines the appeal to (endogenously processed) weights with the use of newly defined scores in Step 3.

\section{Complete analysis of an example}

In order to illustrate the application of our novel algorithm, let us now reconsider the decision problem posed by the fuzzy soft set $(S, P)$ represented by Table 1 .

Example 2 Herrero's $6 \times 6$ Comparison matrix associated with $(S, P)$, whose tabular representation 
has $6 \times 7$ size, is

$$
\left(\begin{array}{rrrrrr}
18 & 1 & 1 & 3 & 4 & 3 \\
3 & 22 & 0 & 3 & 3 & 2 \\
5 & 2 & 24 & 3 & 3 & 3 \\
3 & 2 & 2 & 17 & 1 & 1 \\
3 & 4 & 4 & 5 & 23 & 4 \\
3 & 4 & 4 & 4 & 1 & 22
\end{array}\right)
$$

\begin{tabular}{|c|c|c|c|c|}
\hline & $p_{1}$ & $p_{2}$ & $p_{3}$ & $p_{4}$ \\
\hline$o_{1}$ & 0.6 & 0.7 & 0.3 & 0.3 \\
$o_{2}$ & 0.8 & 0.6 & 0.5 & 0.3 \\
$o_{3}$ & 0.7 & 0.5 & 0.5 & 0.4 \\
\hline
\end{tabular}

Table 2: Tabular representation of the fuzzy soft set $\left(S^{\prime}, P^{\prime}\right)$ in Example 3.

Its dominant eigenvalue is simply $q(k-1)=35$. An eigenvector associated with this eigenvalue is $H=(81.56,86.42,143.46,54.57,159.29,118.60)$, which is unique up to normalization. Hence Algorithm 2 proposes the prioritization

$$
o_{5} \succ o_{6} \succ o_{3} \succ o_{2} \succ o_{1} \succ o_{4}
$$

because $\mathrm{H}_{5}>\mathrm{H}_{6}>\mathrm{H}_{3}>\mathrm{H}_{2}>\mathrm{H}_{1}>\mathrm{H}_{4}$.

This outcome coincides with the solution by Alcantud [5] and is pretty similar to the solution by Algorithm 1 (cf., Example 1).

\section{Comparison with other methodologies}

Example 2 proves that our new proposal (Algorithm 2 ) is different to the solution offered by Roy and Maji [2] (Algorithm 1).

One should also compare it with the variation of the Roy and Maji's approach given by Kong et al.'s [3] controversial algorithm, or with the adjustable approach by Feng et al. [4]. To this purpose it suffices to compare the solutions that they suggest for the problem posed by the fuzzy soft set $(S, P)$ with the outcome of our Algorithm 2. We have calculated that Kong et al.'s [3] algorithm establishes the prioritization $o_{5} \succ o_{3} \succ o_{6} \succ o_{2} \succ o_{4} \succ o_{1}$. By contrast Feng et al.'s solution by their top-level decision rule produces a less clear distinction, namely, $O_{5} \succ o_{3} \succ o_{6} \sim o_{4} \sim o_{2} \sim o_{1}$ (cf., [4, Table 11]). Thus a simple inspection shows that they are different to the solution endorsed here, namely, $o_{5} \succ o_{6} \succ o_{3} \succ o_{2} \succ o_{1} \succ o_{4}$. This argument proves that our Algorithm 2 coincides neither with Kong et al.'s solution nor with Feng et al.'s solution by their top-level decision rule.

The question remains whether the algorithm in Alcantud [5] and Algorithm 2 always provide the same solution (as happened in the particular case of Example 2) or these algorithms are in fact different. In order to solve this question in the negative we provide the following example:

Example 3 Consider the fuzzy soft set $\left(S^{\prime}, P^{\prime}\right)$ represented by Table 2. Its tabular representation has $3 \times 4$ size.

Herrero's $3 \times 3$ Comparison matrix associated with $\left(S^{\prime}, P^{\prime}\right)$ is

$$
\left(\begin{array}{lll}
3 & 1 & 1 \\
2 & 6 & 2 \\
3 & 1 & 5
\end{array}\right)
$$

An eigenvector associated with its dominant eigenvalue $4(3-1)=8$ is $H=(1,3,2)$, which is unique up to normalization. Hence Algorithm 2 proposes the prioritization

$$
o_{2} \succ o_{3} \succ o_{1}
$$

because $\mathrm{H}_{2}>\mathrm{H}_{3}>\mathrm{H}_{1}$.

This outcome does not coincide with the solution by Alcantud [5]. Following the steps in his algorithm one can calculate that the corresponding Comparison matrix is

$$
A=\left(\begin{array}{rrr}
0 & -0.107 & 0.286 \\
0.325 & 0 & 0.068 \\
0.65 & 0.45 & 0
\end{array}\right)
$$

Now it is also immediate to compute the required amounts that provide the scores according to the mechanism in Alcantud [5], namely, $S_{1}=-0.796$, $S_{2}=0.05$ and $S_{3}=0.746$.

Hence Alcantud [5] proposes the prioritization

$$
o_{3} \succ o_{2} \succ o_{1}
$$

which compared with the outcome of Algorithm 2 proves that these two proposals are indeed different. In fact this example proves that they may even disagree on the top alternative when the problem is relatively small.

\section{Conclusion}

We have introduced a recent methodology from Social Choice that provides a new algorithm for solving fuzzy soft set based decision making problems. It can be combined with both Roy and Maji's [2] and Alcantud's [5] methodologies for producing resultant fuzzy soft sets in problems with multiobserver input parameter data set. We have proved by examples that our algorithm is indeed different to existing solutions.

We believe that the tool that we have imported can be succesfully implemented in other related decision making problems in the future. For example, in combination with scores it can be used to provide a ranking of hesitant fuzzy sets over finite sets, which permits useful applications like prioritizing projects. A first attempt with an application to metarankings of world universities appears in Alcantud et al. [24]. 


\section{Acknowledgements}

The author is grateful to Rocío de Andrés Calle for valuable discussions. The author acknowledges financial support by the Spanish Ministerio de Economía y Competitividad (Project ECO201231933).

\section{References}

[1] C. Herrero. An endogenous scoring rule. Mimeo, 2014.

[2] A.R. Roy and P.K. Maji. A fuzzy soft set theoretic approach to decision making problems. Journal of Computational and Applied Mathematics, 203:412-418, 2007.

[3] Z. Kong, L. Gao, and L. Wang. Comment on "A fuzzy soft set theoretic approach to decision making problems". Journal of Computational and Applied Mathematics, 223:540-542, 2009.

[4] F. Feng, Y.B. Jun, X. Liu, and L. Li. An adjustable approach to fuzzy soft set based decision making. Journal of Computational and Applied Mathematics, 234:10-20, 2010.

[5] J.C.R. Alcantud. A novel algorithm for fuzzy soft set based decision making from multiobserver input parameter data set. Mimeo, 2014.

[6] L. Zadeh. Fuzzy sets. Information and Control, 8:338-353, 1965

[7] D. Molodtsov. Soft set theory - first results. Computers and Mathematics with Applications, 37:19-31, 1999.

[8] J.C.R. Alcantud. Some fundamental relationships among soft sets, fuzzy sets, and their extensions. Mimeo, 2014.

[9] P.K. Maji, R. Biswas, and A.R. Roy. Soft set theory. Computers and Mathematics with Applications, 45:555-562, 2003.

[10] H. Aktaş and N. Çağman. Soft sets and soft groups. Information Sciences, 177:2726-2735, 2007.

[11] M.I. Ali. A note on soft sets, rough soft sets and fuzzy soft sets. Applied Soft Computing, 11:3329-3332, 2011.

[12] Feng Feng, Changxing Li, B. Davvaz, and M.Irfan Ali. Soft sets combined with fuzzy sets and rough sets: a tentative approach. Soft Computing, 14(9):899-911, 2010.

[13] Feng Feng, Xiaoyan Liu, Violeta LeoreanuFotea, and Young Bae Jun. Soft sets and soft rough sets. Information Sciences, 181(6):1125 - 1137, 2011.

[14] P.K. Maji, R. Biswas, and A.R. Roy. Fuzzy soft sets. Journal of Fuzzy Mathematics, 9:589-602, 2001.

[15] F. Wang, X. Li, and X. Chen. Hesitant fuzzy soft set and its applications in multicriteria decision making. Journal of Applied Mathematics, pages Article ID 643785, 10 pages, 2014.
[16] B.-H. Han, Y.-M. Li, J. Liu, S.-L. Geng, and H.-Y. Li. Elicitation criterions for restricted intersection of two incomplete soft sets. Knowledge-Based Systems, 59:121-131, 2014.

[17] Yan Zou and Zhi Xiao. Data analysis approaches of soft sets under incomplete information. Knowledge-Based Systems, 21(8):941 - 945, 2008.

[18] Yuncheng Jiang, Yong Tang, Qimai Chen, Hai Liu, and Jianchao Tang. Extending fuzzy soft sets with fuzzy description logics. KnowledgeBased Systems, 24(7):1096 - 1107, 2011.

[19] P.K. Maji, R. Biswas, and A.R. Roy. An application of soft sets in a decision making problem. Computers and Mathematics with Applications, 44:1077-1083, 2002.

[20] Naim Çağman and Serdar Enginoğlu. Soft set theory and uni-int decision making. European Journal of Operational Research, 207(2):848 855, 2010.

[21] Qinrong Feng and Ying Zhou. Soft discernibility matrix and its applications in decision making. Applied Soft Computing, 24:749 - 756, 2014.

[22] F. Feng and Y. Li. Soft subsets and soft product operations. Information Sciences, 232:4457, 2013.

[23] Yuncheng Jiang, Yong Tang, Qimai Chen, Ju Wang, and Suqin Tang. Extending soft sets with description logics. Computers 83 Mathematics with Applications, 59(6):2087 - 2096, 2010.

[24] J.C.R. Alcantud, R. de Andrés Calle, and M.J.M. Torrecillas. Hesitant Fuzzy Worth: an innovative ranking methodology for hesitant fuzzy subsets. Mimeo, 2015. 